\title{
THE EFFECT OF METEOROLOGICAL FACTORS ON ROAD TRAFFIC INJURIES IN BEIJING
}

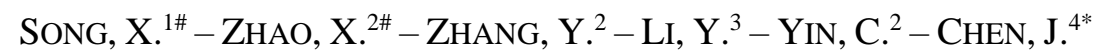

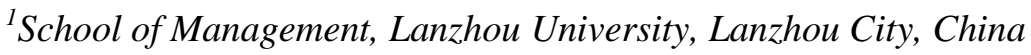 \\ ${ }^{2}$ Department of Neurology, The General Hospital of the People's Liberation Army, Beijing, \\ China \\ ${ }^{3}$ Department of Neurology, The First Affiliated Hospital of Zhengzhou University, Zhengzhou, \\ China \\ ${ }^{4}$ Health Bureau, Logistics Support Department of the Central Military Commission, Beijing, \\ China \\ ${ }^{\#}$ These authors have contributed equally to this research and should be considered as co-first \\ authors. \\ *Corresponding author \\ e-mail: gafm71@163.com
}

(Received $16^{\text {th }}$ Apr 2019; accepted 22 $2^{\text {nd }}$ May 2019)

\begin{abstract}
The goal of this study is to establish the relationship between RTI and the meteorological factors, and make a precise prediction of the occurrence of RTI in China. The statistical data was collected from four tertiary hospitals in Beijing from 2008 to 2012. The association was analyzed using a backward propagation-artificial neural network model (BP-ANN) regression model (built by Matlab) which has three layers (including one hidden layer). Based on the SPSS 20.0 platform, regression analysis was used to evaluate the effect of meteorological factors on RTI. The results show that the most significant factors are atmospheric pressure, temperature, precipitation and sunshine duration, whereas wind speed is less significant. The combination of long sunshine duration, high temperature, low pressure and high humidity is the high-risk condition that leads to RTI. The coefficient $(r=0.7199)$ obtained by the PB neural network is much higher than the coefficient $(r=0.427)$ obtained by the Stepwise Regression Model. Meteorological factors have a certain effect on traffic injury severity. And the BPANN model is a quite precise prediction model for RTI, and this research can provide technical support for the improvement of forecasting and early warning of RTI, in further.

Keywords: risk factors, traffic accident, regression analysis, artificial neural network model, risk warning
\end{abstract}

\section{Introduction}

Road traffic injuries (RTI) are a major global public security issue (Toroyan, 2009). According to a recent report of the World Health Organization (WHO) in May 2015, 1.24 million people are killed by RTIs annually all over the world. This is the leading cause of death in the 15-29 age group. About $91 \%$ of the deaths occur in the low and middle-income countries, while these countries only occupy half of vehicles in the world. It is estimated that RTI will cause 1.9 million deaths from fatal accidents in 2020, and RTI will become the fifth leading cause of death in 2030 (World Health Organization, 2015). For China, the largest developing country in the world, the annual number of deaths caused by RTI increased from about 50,000 in the mid-1980s to more than 100,000 at present (Zhang, et al., 2007; Dong and Peng, 2012). The mortality of 
China is very moderate compared to the average value of world. However, China has the largest population in the world, which makes the death toll huge and result in heavy disease burden and enormous medical expenditure. RTI have become one of the leading causes of unintentional death in China (Gao et al., 2013). Beijing, as the capital of China, has the largest number of vehicles in the country, so, the research about RTI is indispensable.

Thus far, research on RTI focused primarily on human factors, mechanical factors, and road facilities (Evans, 2001; Bendak, 2005; Nakahar et al., 2005; Javouhey et al., 2006; Morgan and Mannering, 2011). However, meteorological factors have received little attention. Nevertheless, the occurrence of RTI is the combined result of human factors, mechanical factors, road facilities and meteorological factors. The change of meteorological factor will have an effect on the other three, leading to the occurrence of RTI. Meteorological factors can cause RTI by affecting mental and psychological state, reaction time, vision, vehicle flow and speed, as well as road conditions (Majdzadeh et al., 2008; Abe et al., 2008; Naci et al., 2009; Gill and Goldacre, 2009; Cools and Moons, 2010; Akin et al., 2011; Kim et al., 2012; Kashani et al., 2012; Grjibovski et al., 2013). For example, precipitation can make road slippery, reduce the friction between vehicles and roads, and increase the difficulty of operating vehicles. Rainfall can reduce the clarity of the windshield of the vehicle. Rainy days will also cause poor visibility of the surrounding environment, affecting both drivers' and pedestrians' vision. Therefore, it is necessary to study the influence of meteorological factors on RTI for effective prevention of traffic injury.

Based on statistical data collected from four tertiary ${ }^{1}$ hospitals in Haidian District, Beijing from 2008 to 2012, the effect of gender, age, date and the effect of meteorological factors were studied using regression analysis in order to determine the main influencing factors on RTI. These factors were used as the predictors in the construction of a backward propagation-artificial neural network model (BP-ANN) with Matlab. BP-ANN is one of the most successful algorithms for the machine learning study which is originally inspired by modeling biological neural systems (more details in the "Model structure" section). Stephan (Stephan et al., 2003) used BP-ANN to create the risk evaluation to prostate cancer. In the study it was found that the ANN technique can be applied to the problems which cannot be solved by any other effective way, and it is particularly suited to evaluate the risk of cancer. In fact, the BP-ANN model can not only used to evaluate the disease, but also to some other significant field. Therefore, we introduced BP-ANN here to make a precise prediction of the occurrence of RTI and provide technical support for decreasing RTI and reducing economic loss.

\section{Resources and methods}

\section{Resources}

In this study, statistical data of 1825 groups from Jan $1^{\text {st }}, 2008$ to Dec $31^{\text {st }}, 2012$ were collected from four tertiary hospitals in Haidian District, Beijing. The data of each group consist of the number of RTI, pressure, humidity, temperature, sunshine duration, rainfall and wind speed in Haidian District. Those meteorological data mentioned above

\footnotetext{
${ }^{1}$ Tertiary hospital is the highest level of Chinese hospital grade, with more than 501 beds, and provides high-level specialist medical services, performs higher education and scientific research tasks in several regions.
} 
were provided by the National Scientific Data Sharing Platform for Population and Health. All the collected data were divided and analyzed according to three dimensions: the age and gender of each injured person, weekly number of RTI, and the value of the corresponding meteorological data (average values for each week).

\section{Regression analysis}

Based on the SPSS 20.0 platform, regression analysis was used to evaluate the effect of meteorological factors on RTI. Major influencing factors were determined through the comparison of the Pearson correlation coefficient $(r)$ and its statistical significance $(p)$ between meteorological factors and RTI. Moreover, the correlation between RTI and the different meteorological factors (such as temperature, relative humidity, pressure, precipitation, sunshine time and wind speed, respectively) were analyzed to determine the main influencing factors.

\section{Construction of the BP-ANN model}

\section{Data set}

All the data were used to construct the BP-ANN model with Matlab. 195 randomly selected groups were used as the training data set and the other 65 groups were used as the test data to evaluate the performance of the optimal BP-ANN model. The large difference between the dimension and the data values of the meteorological factors might affect the forecasting performance of the BP-ANN model. Therefore, the meteorological factors were employed as the independent variables, including atmospheric pressure, atmospheric temperature, precipitation and sunshine duration. And the gaps of each level of these independent variables in the model were $5 \mathrm{hPa}$, $5{ }^{\circ} \mathrm{C}, 2 \mathrm{~mm}$ and $2 \mathrm{~h}$ respectively. Meteorological parameters are input variables as while as the weekly RTI are output variables.

\section{Model structure}

Three parts mainly composed of BP-ANN model: an input layer, a hidden layer and an output layer. There are several nodes in each layer mapping the input vector (in the input layer) to the output vector (in the output layer) by a continuingly updating weight vector (in hidden layer). The weight is updated by each iteration error (the difference between the output value and the real value). The model iterates until the prediction matches the real scenario. BP-ANN is a data-based research method. It uses real world data as the input training set to predict output values in the future without any human experiences.

In our study, the data in the input layer (4 5 neurons) were selected from those meteorological factors which directly or indirectly affect RTI, based on the regression analysis. A suitable number of hidden neurons should be determined during model construction. The number of RTI was chosen as the output layer in the BP model.

The tansig and purelin were selected as transfer functions in the hidden layer and output layer, respectively. The traingdx, an adaptive training function with momentum, was used for network training. This algorithm is able to adjust the learning rate and the incidental momentum automatically, avoiding local minima. Moreover, it accelerates the convergence rate considerably (Shi et al., 2009). 


\section{Evaluation of model performance}

We used the mean squared error (MSE), one of the most widely used evaluation indices of model performance between the monitored and predicted values, to test the prediction accuracy of our BP-ANN model: smaller values of the MSE represent better performance of the model. Another common evaluation index is the correlation coefficient $(r)$, a measure of the relationship between monitored and predicted values. A higher $r$ indicates better performance of the BP-ANN model.

\section{Results and discussion}

\section{Influence of different factors on RTI}

\section{Influence of gender and age on RTI}

Summary statistics of the age and gender distribution of outpatients from 2008 to 2012 (1,827 days) are shown in Table 1. In total, 6,295 hospital admissions for RTI were recorded, and the value was the statistical description of daily value of the patient demographics.

Table 1. Summary statistics of the age and gender distribution of outpatients

\begin{tabular}{c|c|c|c|c|c|c|c}
\hline \multicolumn{2}{c|}{ Term } & Mean & SD & Min & Median & Max & IQR \\
\hline \multirow{3}{*}{ Gender } & Male & 2.10 & 1.48 & 0 & 2 & 10 & 2 \\
& Female & 1.35 & 1.28 & 0 & 1 & 20 & 2 \\
& $1-17$ & 0.29 & 0.15 & 0 & 1 & 4 & 1 \\
\multirow{4}{*}{ Age } & $18-40$ & 1.68 & 0.97 & 0 & 3 & 10 & 5 \\
& $41-65$ & 1.25 & 0.83 & 0 & 3 & 13 & 4 \\
& $>65$ & 0.22 & 0.12 & 0 & 1 & 11 & 2 \\
\hline
\end{tabular}

SD: standard deviation, IQR: inter quartile range

It is seen that there were approximately 4 RTI per day, on average. The number of male traffic injury patients (2.10 cases per day) was larger than that of female $(1.35$ cases per day). The ratio of male to female is 1.56:1. In the 1-17 age group, 0.29 cases of traffic injury occurred, on average, every day. In the 18-40, 41-65 and 65 or over age groups, the daily traffic injury cases were, on average, $1.68,1.25$ and 0.22 , respectively. The maximum mean value of the youth group is 1.35 times that of the adult group, 5.79 times that of the adolescent group and 7.64 times that of the aged group. The result is similar to what was reported by $\mathrm{Yu}$ and Wang et al. (2004), who discovered that young male adults, as the primary labor force, are more exposed to traffic injury. The relationship between these meteorological factors and RTI will be analyzed in next.

\section{Influence of the days of the week and of mental state}

The variation of the number of RTI is indicated in Table 2. From 2008 to 2011, the number of outpatients was rising and the growth rate was $9.73 \%, 9.20 \%$ and $4.40 \%$, respectively. Comparing with the figures corresponding to the periods 2008-2009 and 2009-2010, during the period 2010-2011 RTI decreased sharply. Until 2012, the number of outpatients had fallen to 1125 cases and the decline rate was $27.38 \%$. The reason 
might be that the "vehicle purchase restriction policy" introduced in Beijing in 2011, which greatly reduced the private car purchases. On Monday, Wednesday, Saturday and Sunday, the average total number of outpatient visits is similar: 182, 186, 179, 183 cases respectively. The number of RTI patients is $2.5 \%$ higher on Sunday than that of Saturday, however, the chi-square test showed that the difference is statistical meaningless. In contrast, it reached a peak (202 cases) on Friday and a valley (163 cases) on Tuesday. The data above were divided into 2 parts. One was from Monday to Thursday and the other was from Friday to Sunday. The corresponding averages were 173.6 and 188.2, respectively. This is similar to the result of "a week working rhythm", by D. S. Moskowitz. The research concluded that people tend to be more energetic at the beginning of the week and then gradually get fatigued. Especially on Tuesday, people are more controlled, efficient and concentrated on work. In contrast, they are more reckless on Friday. It is reported that on Friday, drivers hasten to participate in entertainment activities and alcohol consumption is higher. This leads to a $13 \%$ higher occurrence rate of RTI.

Table 2. Comparison of RTI on different days in one week

\begin{tabular}{c|c|c|c|c|c|c|c|c}
\hline Year & Mon & Tue & Wed & Thu & Fri & Sat & Sun & Total \\
\hline 2008 & 167 & 147 & 171 & 157 & 184 & 145 & 152 & 1123 \\
2009 & 155 & 135 & 197 & 187 & 201 & 184 & 185 & 1244 \\
2010 & 202 & 205 & 187 & 158 & 227 & 194 & 197 & 1370 \\
2011 & 216 & 176 & 213 & 178 & 236 & 209 & 205 & 1433 \\
2012 & 169 & 150 & 160 & 142 & 164 & 162 & 178 & 1125 \\
Average & 181.8 & 162.6 & 185.6 & 164.4 & 202.4 & 178.8 & 183.4 & 1259 \\
\hline
\end{tabular}

The influence of drinking and medicine should be considered in this study. But according to the information gathered from the emergency doctors who collected the data, the RTI caused by drunk driving only occupies the proportion very few. Meanwhile, Saha et al. (2016) pointed out that the odds of risk factors (alcohol, drug involvement and so on) were low, among these fatalities associated with adverse weather conditions. So it can be considered that the effect of drinking and medicine are minimal.

\section{Determination of critical meteorological factors}

To study the effect of meteorological factors on RTI in Beijing, the Spearman Correlative Analysis Method was employed. It was found that performance was superior when weekly data was used. Comparing to daily data (level of meteorological factor), it was able to eliminate strong "Weekly Effect" of RTI. The correlation among RTI, pressure, temperature, relative humidity, precipitation, sunshine duration and wind speed, on weekly basis, is shown in Table 3.

It was shown that RTI have a strong positive correlation with temperature, precipitation and sunshine duration and were negatively correlated with pressure. No correlation of statistical significance with wind speed was obtained.

According to Hani et al. (2009), as temperature increases, the sensitivity of body decreases, the heart rate increases and the driving and physical capacity decrease. Moreover, temperature increase could cause debility and that could seriously affect 
drivers (Cool and Moons, 2010; Keay and Simmonds, 2005). As reported previously (Brijs et al., 2006; Bossche and Wets, 2005), temperature is positively correlated with vehicle flow and speed, which are the important factors in RTI. This research showed that RTI are positively correlated with sunshine duration, as in Brijs et al. (2006). A longer sunshine duration will lead to the increases of air temperature. On the one hand, high atmospheric temperature will induce drivers' persistent excitability of nervous system; they will get tired more easily than usual. On the other hand, the longer sunshine show that the daytime is longer than night time, people prefer to drive on the daytime. Therefore, RTI increase accordingly.

Table 3. Spearman correlations between RTI and meteorological factors

\begin{tabular}{c|c|c|c|c|c|c|c}
\hline & RTI & $\begin{array}{c}\text { Temperature } \\
\left({ }^{\circ} \mathrm{C}\right)\end{array}$ & $\begin{array}{c}\text { Relative } \\
\text { humidity }(\%)\end{array}$ & $\begin{array}{c}\text { Pressure } \\
(\mathbf{h P a})\end{array}$ & $\begin{array}{c}\text { Precipitation } \\
(\mathbf{m m})\end{array}$ & $\begin{array}{c}\text { Sunshine time } \\
(\mathbf{h})\end{array}$ & $\begin{array}{c}\text { Wind speed } \\
(\mathbf{m} / \mathbf{s})\end{array}$ \\
\hline RTI & 1.000 & & & & & & \\
Temperature $\left({ }^{\circ} \mathrm{C}\right)$ & $0.103^{* *}$ & 1.000 & & & & & \\
Relative humidity $(\%)$ & $0.033^{* *}$ & $0.394^{* *}$ & 1.000 & & & & \\
Pressure (hPa) & $-0.079^{* *}$ & $-0.872^{* *}$ & $-0.375^{* *}$ & 1.000 & & & \\
Precipitation (mm) & $0.043^{* *}$ & $0.199^{* *}$ & $0.471^{* *}$ & $-0.258^{* *}$ & 1.000 & & \\
Sunshine time (h) & $0.084^{* *}$ & $0.175^{* *}$ & $-0.561^{* *}$ & $-0.084^{* *}$ & $-0.363^{* *}$ & 1.000 & \\
Wind speed $(\mathrm{m} / \mathrm{s})$ & 0.036 & $-0.026^{*}$ & $-0.477^{* *}$ & 0.013 & $0.025^{*}$ & $0.265^{* *}$ & 1.000 \\
\hline
\end{tabular}

$* P<0.05 ; * * P<0.01$

In this study, it is shown that there is a positive correlation between the precipitation and RTI $(\mathrm{OR}=0.148, p<0.05)$. Precipitation can reduce the friction coefficient, the visibility of the surroundings and the sharpness of the windshield (Qiu and Nixon, 2008). Moreover, it may influence the judgment of drivers and pedestrians (Andrey, 2010). Wang et al. (2014) pointed out that rain and snow are the two leading causes of traffic accidents in Gansu province, China. They account for $97.58 \%$ of the traffic accidents caused by meteorological causes. Some studies have confirmed that precipitation is an important risk factor (Bendak, 2005; Zhang et al., 2000; Warrell and Univ, 2012), whereas others assert that rain may in fact reduce the risk of car accidents, as drivers are forced to drive at lower speeds (Hani et al., 2009; Zhang et al., 2000). This may be attributed to regional differences.

In this study, it is shown that the number of outpatients is negatively correlated with pressure. Warrell and others found that the emotions of people and animals could be affected by low pressure, with symptoms such as chest distress, shortness of breath and irritability (Warrell and Univ, 2012; Franka and Rupprecht, 2011; Alice et al., 2011). The combination of high temperature, low pressure, and high humidity has a negative effect on physical capacity; therefore drivers should be particularly attentive to the pedestrians.

In any event, atmospheric pressure, temperature, precipitation and sunshine duration may be considered as the major meteorological factors that influence RTI in Beijing.

\section{Prediction of RTI using a BP-ANN model}

An increase of the number of hidden neurons can improve prediction accuracy. However, this leads to a longer learning process and reduced ability for generalization (May and Sivakumar, 2009; Sadrzadeh et al., 2009). In contrast, a decrease in the number of hidden neurons will result in smaller fault tolerance and capacity to identify 
the new data that will be imported (Maier and Jain, 2010). Thus, the number of hidden neurons should be suitably chosen in order to enhance the prediction accuracy of the BP-ANN model. In this paper, a comparison of the value of the MSEs of the true value and the forecasting value of the test data with different number of hidden neurons (3 12) in the hidden layer was made, in order to obtain the optimal performance of the BP-ANN model. The result is shown as Figure 1. When the number of neurons was 6 in the hidden layer, the minimum MSE (which was obtained after 30 parallel calculations) of the true and the forecasting value was 0.48 . This signified optimal performance of the BP-ANN model.

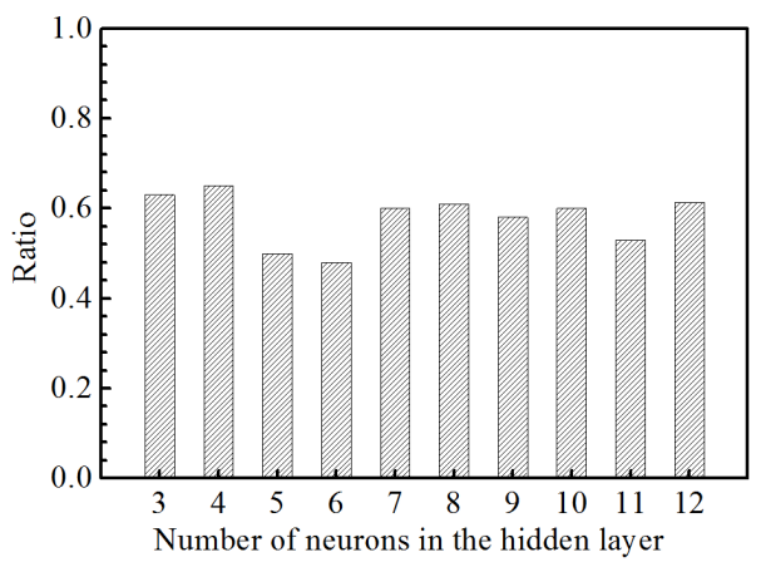

Figure 1. Ratio of MSEs of the true value to forecasting value vs. different number of neurons in the hidden layer

Figure $2 a$ and $b$ show the relationship between the true value and the forecasting value of the test data with optimal performance of the BP-ANN model. Since our investigation of the RTI was conducted in Beijing, a relatively high coefficient $(r=0.7199)$ was obtained. This is much higher than the coefficient $(r=0.427)$ obtained using the Stepwise Regression Model (Ji, 2015). Thus, the BN-ANN model constructed in this work was capable of making an effective prediction of the RTI when using the average pressure, temperature, rain and sunshine duration as the input data.
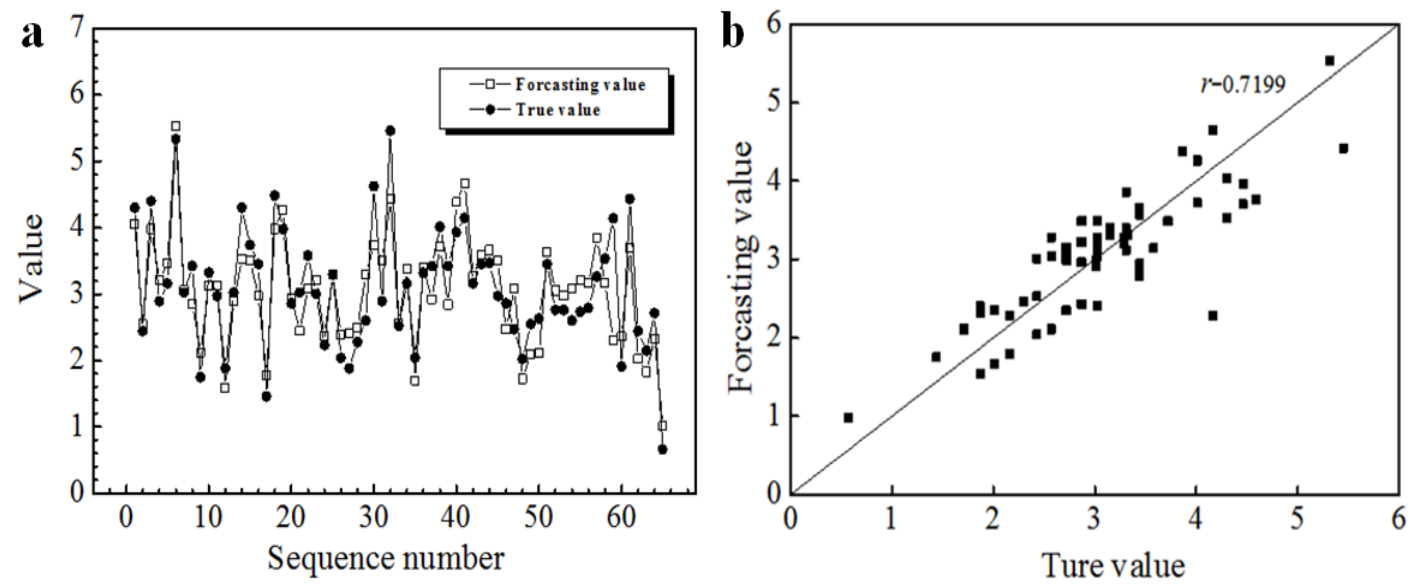

Figure 2. Regression plots (a) and comparison (b) of the true and forecasting values of the test data of $R T I$ 
Moreover, the model performed better when it was used for the prediction of RTI in the interval between 2 to 4 . This is probably due to the relatively limited data set and the small interval used for the construction of the BP-ANN model. Therefore, effort should be made to supply more data to the prediction model.

\section{Conclusions}

In this study, A BP-ANN regression model is investigated to analyze the relationship between the meteorological factors and RTI. The main conclusions can be summarized as below:

1) Young males (18 to 40 years old) own the highest probability of occurrence of RTI; and Friday is day with highest frequency of RTI in a week.

2) Furthermore, meteorological factors have a certain effect on traffic injury severity. The most significant factors are atmospheric pressure, temperature, precipitation and sunshine duration, whereas wind speed is less significant. The combination of long sunshine duration, high temperature, low pressure and high humidity is the high-risk condition that leads to RTI.

3) It can be concluded that the BP-ANN model is a quite precise prediction model for RTI, Furthermore, this research can provide technical support for the improvement of forecasting and early warning of RTI, in further.

Acknowledgements. This research is supported by the National Key R\&D Program of China (2017YFC1307700).

\section{REFERENCES}

[1] Abe, T., Tokuda, Y., Ohde, S., Ishimatsu, S., Nakamura, T., Birrer, R. B. (2008): The influence of meteorological factors on the occurrence of trauma and motor vehicle collisions in Tokyo. - Emergency Medicine Journal 25(11): 769-772.

[2] Akin, D., Sisiopiku, V. P., Skabardonis, A. (2011): Impact of weather on traffic flow characteristics of urban freeways in Istanbul. - Procedia - Social and Behavioral Sciences 16: 89-99.

[3] Alice, L., Green, L., Dunn, J. R. (2011): Rabies epidemiology, risk assessment, and preand post exposure vaccination. - The Veterinary Clinics of North America. Exotic Animal Practice 14(3): 507-518.

[4] Andrey, J. (2010): Long-term trends in weather-related crash risk. - Journal of Transport Geography 18: 247-258.

[5] Bendak, S. (2005): Seat belt utilization in Saudi Arabia and its impact on road accident injuries. - Accident Analysis \& Prevention 37: 367-371.

[6] Bossche, F., Wets, G. (2005): Role of exposure in analysis of accidents: a Belgian case study. - Journal of the Transportation Research Board 1908: 96-103.

[7] Brijs, T., Offermans, C., Hermans, E. (2006): Impact of weather conditions on road board safety investigated on hourly basis. - Transportation Research Board 85th Annual Meeting, Washington, DC.

[8] Cools, M., Moons, E. (2010): Assessing the impact of weather on traffic intensity. Weather Clim Soc 2: 60-68.

[9] Dong, X. M., Peng, L. (2012): The development of intervention on traffic accident injury. - Chinese Journal of Public Health 28: 569-571. 
[10] Evans, L. (2001): Female compared with male fatality risk from similar physical impacts. - The Journal of Trauma 50: 281-288.

[11] Franka, R., Rupprecht, C. E. (2011): Treatment of rabies in the 21st century: curing the incurable. - Future Microbiol 6(10): 1135-1140.

[12] Gao, J. H., Zhu, Y., Li, L. P. (2013): The association between meteorological factors and traffic injury sufferers admitted to a tertiary hospital in Shantou City. - Inj Med 2: 26-31.

[13] Gill, M., Goldacre, M. J. (2009): Seasonal variation in hospital admission for road traffic injuries in England: analysis of hospital statistics. - Injury Prevention 15(6): 374-378.

[14] Grjibovski, A. M., Kosbayeva. A., Menne, B. (2013): The effect of ambient air temperature and precipitation on monthly counts of salmonellosis in four regions of Kazakhstan, Central Asia, in 2000-2010. - Epidemiology and Infection 2(3): 1-8.

[15] Hani, S., Dong, J., Jiwon, K. (2009): Incorporating Weather Impacts in Traffic Estimation and predication system. - U.S. Department of Transportation, Research and Innovative Technology Administration, Washington, DC.

[16] Javouhey, E., Guerin, A. C., Chiron, M. (2006): Incidence and risk factors of severe traumatic brain injury resulting from road accidents: a population-based study. - Accident Analysis \& Prevention 38(2); 225-233.

[17] Ji, C. H. (2015): Analysis of trauma incidence characteristics in Beijing and its relationship with meteorological conditions. - Lanzhou University 6.

[18] Kashani, A. T., Shariat-Mohaymany, A., Ranjbari, A. (2012): Analysis of factors associated with traffic injury severity on rural roads in Iran. - Journal of Injury \& Violence Research 4(1): 36.

[19] Keay, K., Simmonds, I. (2005): The association of rainfall and other weather variables with road traffic volume in Melbourne, Australia. - Accident Analysis \& Prevention 37(1): 109-124.

[20] Kim, Y., Kim, H., Shin, S. D., Hong, Y. C. (2012): Different influence of outdoor temperature on traumatic and non-traumatic injuries. - Journal of Trauma and Acute Care Surgery 73(4): 944-949.

[21] Maier, H. R., Jain, A. G. (2010): Methods used for the development of neural networks for the prediction of water resource variables in river systems: current status and future directions. - Environmental Modelling and Software 25: 891-909.

[22] Majdzadeh, R., Khalagi, K., Naraghi, K., Motevalian, A., Eshraghian, M. R. (2008): Determinants of traffic injuries in drivers and motorcyclists involved in an accident. Accident Analysis \& Prevention 40: 17-23.

[23] May, D. B., Sivakumar, M. (2009): Prediction of urban storm water quality using artificial neural networks. - Environmental Modelling and Software 24(2): 296-302.

[24] Morgan, A., Mannering, F. L. (2011): The effects of road-surface conditions, age, and gender on driver-injury severities. - Accident Analysis \& Prevention 43: 1852-1863.

[25] Naci, H., Chisholm, D., Baker. TD. (2009): Distribution of road traffic deaths by road user group: a global comparison. - Injury Prevention 15(1): 55-59.

[26] Nakahar, S., Chadbunchachai, W., Ichikawa, M., Tipsuntornsak, N., Wakai, S. (2005): Temporal distribution of motorcyclist injuries and risk of fatalitiesin relation to age, helmet use, and riding while intoxicated in KhonKaen, Thailand. - Accident Analysis \& Prevention 37: 833-842.

[27] Qiu, L., Nixon, W. A. (2008): Effects of adverse weather on traffic crashes: systematic review and meta-analysis. - Transportation Research Record Journal of the Transportation Research Board 2055: 139-146.

[28] Sadrzadeh, M., Mohammadi, T. J., Ivakpour, J., Kasiri, N. (2009): Neural network modeling of $\mathrm{Pb} 2+$ removal from wastewater using electrodialysis. - Chemical Engineering and Processing 48(8): 1371-1381.

[29] Saha, S., Schramm, P., Nolan, A., Hess, J. (2016): Adverse weather conditions and fatal motor vehicle crashes in the United States, 1994-2012. - Environmental Health 15(1): 104. 
[30] Shi, Y., Zhao, X. T., Zhang, Y. M., Ren, N. Q. (2009): Back propagation neural network (BPNN) prediction model and control strategies of methanogen phase reactor treating traditional Chinese medicine wastewater (TCMW). - Journal of Biotechnology 144(1): 70-74.

[31] Stephan, C., Vogel, B., Cammann, H., Lein, M., Klevecka, V., Sinha, P., Kristiansen, G., Schnorr, D., Jung, K., Loening, S. A. (2003): An artificial neural network as a tool in risk evaluation of prostate cancer. Indication for biopsy with the PSA range of 2-20 microg/l. - Der Urologe Ausg A 42(9): 1221-1229.

[32] Toroyan, T. (2009): Global status report on road safety: time for action. - Inj Prev 15(4): 286.

[33] Wang, Y. S., Pu, S., Meng, L. X., Chen, C., Fang, Y. M. (2014): Analysis of meteorological effect of highway traffic accidents in Gansu. - Gansu Science and Technology 44(21): 25-28.

[34] Warrell, M. J., Univ, O. (2012): Current rabies vaccines and prophylaxis schedules: Preventing rabies before and after exposure. - Travel Medicine and Infectious Disease 10(1): 1 .

[35] World Health Organization (2015): The 358th live report of WHO. - World Health Organization, Geneva.

[36] Yu, J. M., Wang, Y. C. (2004): Retrospective Study on Road Related Injury. - Journal of Tong Ji University 12: 513-516.

[37] Zhang, J., Lindsay, J., Clarke, K., Robbins, G., Mao, Y. (2000): Factors affecting the severity of motor vehicle traffic crashes involving elderly drivers in Ontario. - Accident Analysis and Prevention 32(1): 117-125.

[38] Zhang, X. J., Chen, Z. D., Hao, Y. C., Gao, S. X., Chen, Y. Q. (2007): Analysis of road traffic accident injury from 1951 2014 in China. - Chinese Journal of Public Health 23(10): 1214-1215. 